\title{
Reactor Start-up and Control Methodologies: Consideration of the Space Radiation Environment
}

\author{
Shannon M. Bragg-Sitton and James Paul Holloway \\ Department of Nuclear Engineering and Radiological Sciences, University of Michigan, Ann Arbor, MI 48109 \\ (256)544-6272; shanbrag@engin.umich.edu
}

\begin{abstract}
The use of fission energy in space power and propulsion systems offers considerable advantages over chemical propulsion. Fission provides over six orders of magnitude higher energy density, which translates to higher vehicle specific impulse and lower specific mass. These characteristics enable the accomplishment of ambitious space exploration missions. The natural radiation environment in space provides an external source of protons and high energy, high $Z$ particles that can result in the production of secondary neutrons through interactions in reactor structures. Initial investigation using MCNPX 2.5.b for proton transport through the SAFE-400 reactor indicates a secondary neutron net current of $1.4 \times 10^{7} \mathrm{n} / \mathrm{s}$ at the core-reflector interface, with an incoming current of $3.4 \times 10^{6} \mathrm{n} / \mathrm{s}$ due to neutrons produced in the Be reflector alone. This neutron population could provide a reliable startup source for a space reactor. Additionally, this source must be considered in developing a reliable control strategy during reactor startup, steady-state operation, and power transients. An autonomous control system is developed and analyzed for application during reactor startup, accounting for fluctuations in the radiation environment that result from changes in vehicle location (altitude, latitude, position in solar system) or due to temporal variations in the radiation field, as may occur in the case of solar flares. One proposed application of a nuclear electric propulsion vehicle is in a tour of the Jovian system, where the time required for communication to Earth is significant. Hence, it is important that a reactor control system be designed with feedback mechanisms to automatically adjust to changes in reactor temperatures, power levels, etc., maintaining nominal operation without user intervention. This paper will evaluate the potential use of secondary neutrons produced by proton interactions in the reactor vessel as a startup source for a space reactor and will present a potential control methodology for reactor startup procedures in the event of source fluctuations.
\end{abstract}

\section{INTRODUCTION}

Space vehicles are exposed to a large amount of radiation including protons, electrons, and atomic nuclei, with energies up to tens of $\mathrm{GeV}$ per nucleon. Contributions to this radiation field include trapped charged particles, galactic cosmic rays (GCR), anomalous cosmic rays (ACR), and particles arising from solar particle events (SPEs). Several models have been generated from experimental data to approximate the populations and energy spectra of each of these components at various times during the 11-year solar cycle. Developed by the Naval Research Laboratory, CREME96 (Cosmic Ray Effects on Micro-Electronics) uses satellite data taken at 1 A.U. and, from this, can generate numeric models of the ionizing radiation environment in near-Earth or geosynchronous orbits (Naval Research Laboratory, 1986), taking into account the complex effects of solar variation. The space radiation environment includes all naturally occurring nuclides, ranging from an atomic number of one to 92 , having energies from less than $1 \mathrm{MeV}$ to as high as $10^{15} \mathrm{MeV}$ (total energy) (Feynman and Gabriel, 1996). The radiation environment at geosynchronous orbit provides a reasonable estimate for a background radiation source during reactor startup. The CREME96 database corresponds to average values of the particle fluence during quiet or stormy geomagnetic conditions, during solar maximum or solar minimum, and for varying levels of solar activity.

The solar cycle has an approximate 11-year period. The period of high solar activity, known as solar maximum, extends approximately seven years. The "quiet" sun period, or solar minimum, extends the remaining four years of the solar cycle. Contrary to what one might expect, the solar minimum period corresponds to higher particle fluence in near-Earth and geosynchronous orbits. During solar maximum, the solar wind is more dynamic and is more effective in impeding GCR penetration into the solar system, resulting in an inverse correlation of the GCR and the level of solar activity. The cyclical variations in the GCR fluence are most pronounced at low particle energies, 
becoming rather insignificant at energies greater than $10 \mathrm{MeV}$ per nucleon. ACR fluence can vary by as much as a factor of 500 from solar minimum to maximum, with the higher fluence being observed during solar minimum (Tylka et al., 1997). During solar events, the solar proton fluence in Earth orbit increases, but the trapped particle radiation flux decreases due to a variation in the magnetic fields and atmospheric expansion. As a sum result of these effects, the trapped proton source can decrease by approximately a factor of two during solar maximum. Atmospheric expansion removes more trapped protons from the radiation belts due to the increased energy loss and scattering in the residual atmosphere.

CREME96 incorporates two models for the geomagnetic "weather" conditions. The "solar quiet" model assumes that all fields are present at nominal levels, with the output reflecting long-term averages of the particle fluence. The "stormy" model assumes that disturbances exist in the magnetosphere, decreasing the magnetic shielding of the Earth and increasing the ability of cosmic rays to access near-Earth space. In addition to geomagnetic conditions, CREME96 provides three different models relating to solar energetic particles. During a solar particle event (SPE), commonly referred to as a solar flare, increased particle fluence is observed in near-Earth space. The models included in CREME96 are based on the SPE that occurred in October 1989. The 1989 event was the largest in recent recorded history, in which instruments were available to record the full energy spectrum of the particles. A larger event was observed in 1956, but detailed information on the particle types and energy spectra for this event were not recorded. Hence, a conservative "worst-case" scenario is generally considered to demonstrate a particle fluence that is 10 times greater than the 1989 event (Wilson et al., 1997).

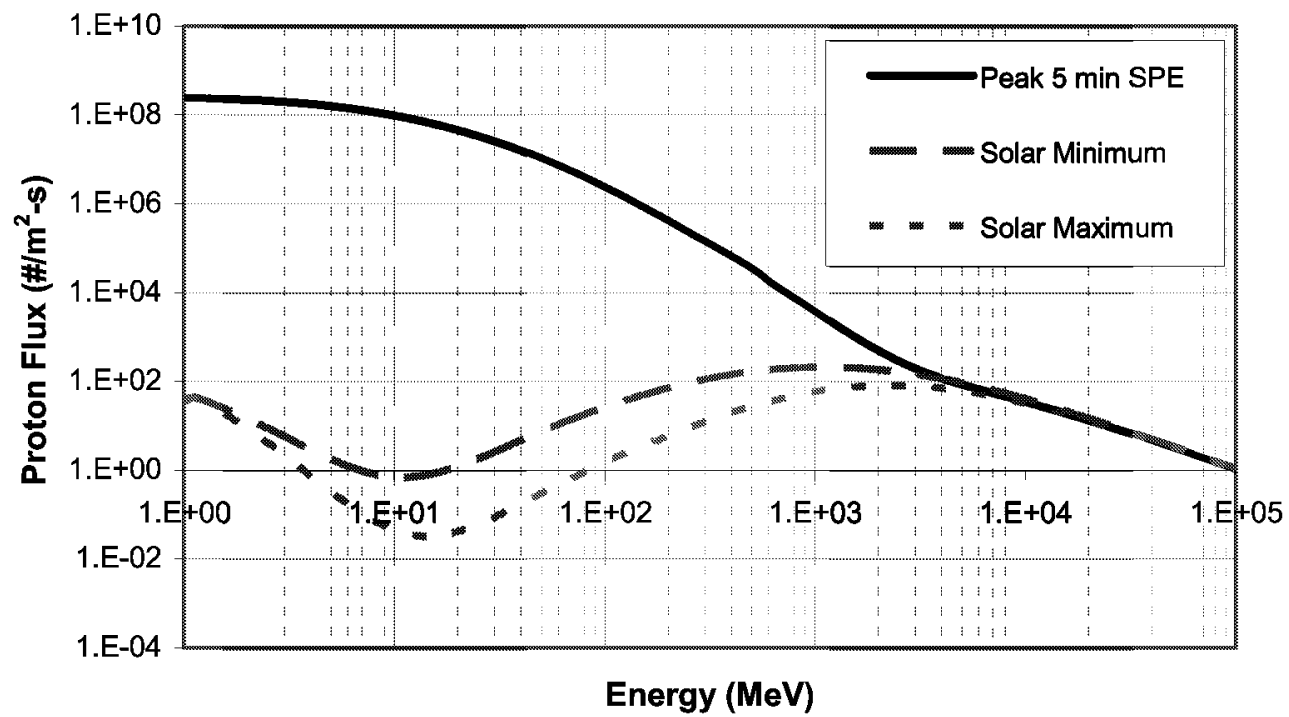

FIGURE 1. Proton Flux in Geosynchronous Earth Orbit, Predicted by CREME96 (Naval Research Laboratory, 1996).

A potential mission for a fission-powered spacecraft utilizing a nuclear electric propulsion system is to the Jovian system. Although the specific reactor design has not yet been selected for such a mission, any of the possible reactor designs would require reactor startup at a nuclear-safe orbit in near-Earth space. The background condition selected for reactor startup was the environment at geosynchronous orbit during stormy geomagnetic conditions and at solar minimum. Minimum source conditions at solar maximum should also be considered to demonstrate that the reactor can be started with any probable background source. An "extreme" environment was characterized by the peak 5-minute flux recorded during the October 1989 event (Naval Research Laboratory, 1996). Fig. 1 shows a comparison of the proton environments during each of these conditions, as well as a comparison to the flux at geosynchronous orbit during solar maximum. In the present analysis, only the proton component of the space radiation field is considered in secondary particle production due to limitations in the MCNPX 2.5.b code. The effect of high $\mathrm{Z}$ particles will be assessed in future analyses. 


\section{Baseline Space Reactor System: SAFE-400}

Energetic protons can cause spallation in the reactor structure, producing a large number of secondary, lighter nuclear species, including neutrons that can initiate a chain reaction in fissionable material. Hence, a subcritical space reactor will have a sustained neutron population.

The SAFE-400 fission reactor, designed at Los Alamos National Laboratory (LANL) for use in unmanned, deep space missions, was used as a baseline reactor design to study the effect of the external radiation environment. SAFE (Safe, Affordable Fission Engine) is a fast spectrum, heatpipe cooled reactor designed to operate at $400 \mathrm{~kW}$ thermal (kWt) (Poston et al., 2002). Reactor control is accomplished by cylindrical control drums housed within the radial reflector that surrounds the reactor core, each containing a section of $\mathrm{B}_{4} \mathrm{C}$ within a $120^{\circ}$ arc. When rotated to the inner position, the $\mathrm{B}_{4} \mathrm{C}$ serves as a reactor poison, absorbing neutrons and preventing the reactor from becoming critical. As the control drums are rotated out, the reactivity is increased and the reactor can become critical. At a drum position of $90^{\circ}$, the effective multiplication is $1.00021 \pm 0.00024$, corresponding to a reactivity of nearly zero.

A complete detailed model of the SAFE-400 reactor was constructed using MCNPX 2.5.b (Waters, 2002). An axial cross section of the reactor model is shown in Fig. 2. To compute the effects of the radiation environment on the neutron population in the reactor, the reactor control drums were positioned such that the system was subcritical $\left(\mathrm{k}_{\text {eff }}\right.$ $=0.97695 \pm 0.00022$ ). The reactor was then bombarded isotropically with the proton flux discussed previously (see Fig. 1) and the resulting neutron population was quantified.

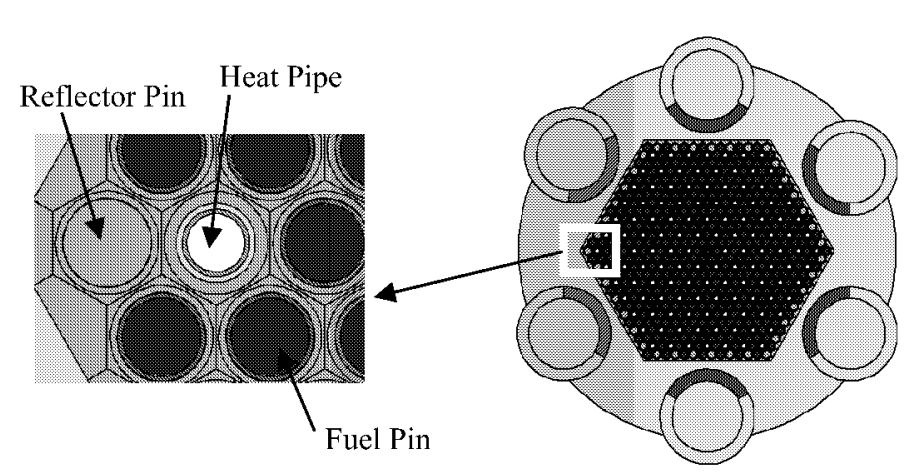

(a) (b)

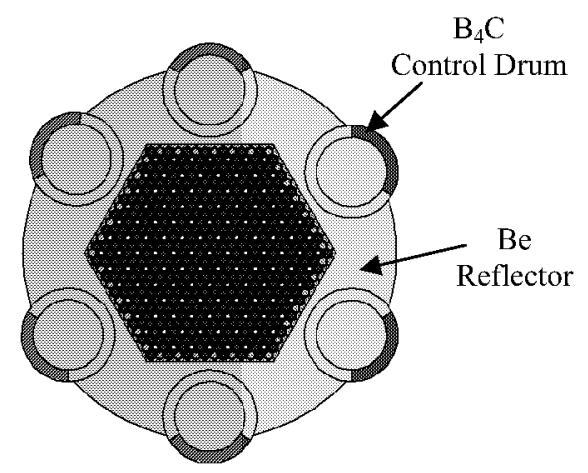

(c)

FIGURE 2. Axial Cross Section of SAFE-400 Core for Control Drum Positions of (b) $\theta=0^{\circ}$ and (c) $\theta=180^{\circ}$.

\section{THEORY: REACTOR CONTROL}

Reliable reactor control is of imminent importance to reactor safety, both in terrestrial and space systems. For a space system, where the time for communication to Earth is significant, autonomous control is imperative. Based on feedback from reactor diagnostics, a controller must be able to automatically adjust to changes in reactor temperature and power level to maintain nominal operation without user intervention. Of particular interest to the present study is the ability of the reactor control system to provide appropriate control to start the reactor from a cold, zero-power condition in the presence of a time-varying external radiation source within a relatively short time. During steady-state operation of a reactor at the nominal power level (e.g., $400 \mathrm{kWt}$ ), the neutron population in the reactor is significantly larger than the neutron population generated by external radiation sources. However, during startup, the reactor neutron population is very low and could be dominated by the secondary neutrons produced by interactions of the high energy protons and high $\mathrm{Z}$ particles in the external environment. The latter operational regime is of interest in the present study.

Model-based predictive control (MBPC) is investigated as a potential control methodology for reactor startup. MBPC is a closed-loop feedback approach that optimizes the control vector over a "receding-horizon" (Clarke, 1994; Morari, 1994). At each sample time, an optimal control vector, $u(t)$, is found over a pre-defined horizon of 
length $h$, but only the first step in the control is actually applied to the plant. Optimization of the objective function over the same horizon is then repeated at the next sample time. Feedback from the current plant condition at each sample interval is determined from measurements; this feedback can be used to "realign" the control at each step, avoiding the accumulated errors that can result from open loop control. The cornerstone of MBPC is the model, and selecting an appropriate model for the system will determine the effectiveness of the controller in plant operation. In addition, MBPC requires that some terminal constraint be applied to reach a desired objective. A terminal equality constraint may be applied such that over an additional range of $h$ points, the predicted plant output will equal the goal set for the controlled parameter.

\section{System Model: Point Kinetics Equations}

The reactor dynamics are modeled using the point kinetics equations (PKE), given as Eqs. (1), which can be derived from transport and diffusion theory (Hetrick, 1971). The most important difference between fast and thermal reactors is the time scale for neutron reproduction, represented in the PKE by the prompt neutron lifetime, A. The PKE representation provides only an approximate model of the reactor; it does not provide a mechanism to describe neutron energy effects or structural details in a heterogeneous reactor. Because fast reactors are relatively small in size and do not contain any moderator to slow down the neutrons to a lower energy, the PKE are a good approximation for the dynamics in a fast reactor. The definitions of each term are provided in the Nomenclature section at the end of this paper.

$$
\begin{gathered}
\frac{d n}{d t}=\frac{\rho-\beta}{\Lambda} n+\sum_{i} \lambda_{i} C_{i}+Q \\
\frac{d C_{i}}{d t}=\frac{\beta_{i}}{\Lambda} n-\lambda_{i} C_{i} .
\end{gathered}
$$

The components of Eq. (1a) represent the neutron production and loss rates from prompt neutrons, delayed neutrons, and the external source. The second balance equation $(\mathrm{lb})$ corresponds to the rate of production and loss of the delayed neutron precursors. Studies of reactor dynamics generally recognize six distinct groups of delayed neutrons. The initial studies of reactor control presented here will consider only one group of delayed neutrons having a decay constant, $\lambda$, equivalent to a weighted average of the six individual decay constants. In the presence of an external source, $Q$, examination of the steady-state solution to the point kinetics equations indicates that a subcritical reactor $\left(k_{e f f}<1\right)$ can be made to be self-sustaining. At steady-state, the reactivity is given by $\rho=-Q / n_{s s}$, where $n_{s s}$ is the neutron population at steady state. Recalling that $\rho=\left(k_{\text {eff }}-1\right) / k_{\text {eff }}$, this result corresponds to $k_{\text {eff }}<1$ when $Q$ is nonzero. Hence, one would expect the final control drum position to correspond to a $k_{e f f}$ that is slightly less than one in the presence of an external source. The reactor neutron population, $n$, can be converted to reactor power, $P$, using the expression in Eq. (2), where $w$ corresponds to the amount of energy produced in a single fission reaction $(w=$ $200 \mathrm{MeV} /$ fission) and $v$ is the number of neutrons produced per fission event.

$$
P=n \frac{w}{\Lambda v} .
$$

If we assume that there is no reactivity feedback present, then the system of differential equations presented in Eqs. (1) is linear in $\mathrm{n}$ and C. However, the plant description is still highly nonlinear in its dependency on the angular position of the control drums, resulting in a nonlinear control problem. If temperature feedback is included in the expression for reactivity, $\rho$ becomes $\rho(n(t))$, and the equations become nonlinear. At low power operation, as during startup, the reactor temperature is relatively low, such that temperature effects do not have a significant effect on the reactivity. Therefore, the present consideration will not include temperature feedback effects on the reactivity. Control to full power operation will require that feedback effects be added in the description of the reactor dynamics. 


\section{Control Methodology}

In the reactor system described, the controlled parameter is the rate at which the control drums are rotated by the control drum drive motors. Given by Eq. (3), the rotation rate yields a time-dependent function for the angular position of the control drums.

$$
\omega(t)=\frac{d \theta(t)}{d t} .
$$

The angular position of the control drums governs the amount of reactivity in the reactor. The reactivity as a function of control drum position $\theta$ (in degrees) was computed using several kcode calculations in MCNPX, with the control drums incremented $30^{\circ}$ in each subsequent run. In all cases, the error in the calculated reactivity was less than $0.04 \%$. A fourth order polynomial was fit to the kcode results using a least squares fit in Microsoft Excel $\left(\mathrm{R}^{2}=\right.$ 1), providing a connection between the control vector and the reactor model.

$$
\rho(\theta)=1.81 \cdot 10^{-4} \theta^{4}-1.00 \cdot 10^{-7} \theta^{3}+1.57 \cdot 10^{-5} \theta^{2}+1.79 \cdot 10^{-4} \theta-4.99 \cdot 10^{-2} .
$$

In MBPC, the present and future control actions are found by solution of an optimization problem. In defining a cost function, $J$, for the optimization, primary consideration is given to the difference between the present reactor power, $P(t)$, and the desired reactor power, $P_{\text {goal }}$. Large changes in the control vector are also penalized to prevent oscillation in the control, improving the stability of the result. An additional penalty, given by the parameter fitheta, is applied if the present iteration in the function minimization selects an $\omega$ leading to a $\theta$ outside the desired range of $0^{\circ}$ to $180^{\circ}$. This penalty provides a simple mathematical representation of a mechanical stop that would likely be present in the control drum drive, preventing the drums from rotating past $180^{\circ}\left(0^{\circ}\right.$ represents the initial drum position prior to startup). The selected cost function is provided in Eq. (5). Appropriate values for the weighting coefficients $a, b$ are selected for each control problem.

$$
J=f \text { theta } \cdot\left[\int_{t_{o}}^{t_{0}+h}\left(P(t)-P_{\text {goal }}\right)^{2} d t\right]+a u^{2}+b(\Delta u)^{2}
$$

MATLAB 6.1 was selected for computation of the optimal reactor control. Minimization of the cost function was accomplished using the fminsearch algorithm within MATLAB's Optimization Toolbox. Fminsearch utilizes a Nelder-Mead simplex algorithm, a direct search method appropriate for multi-dimensional unconstrained nonlinear optimization (Lagarias, et al., 1998), to find the minimum of the indicated function to a prescribed tolerance. In this case, the objective function that is minimized is given by Eq. (5), where the reactor power, $P(t)$, was determined from solution of the PKE given in Eqs. (1). To find a solution to the PKE within the MATLAB framework, a stiff ODE solver, ode1 $5 \mathrm{~s}$, is applied.

\section{RESULTS AND DISCUSSION}

Using MCNPX 2.5.b with a fully specified SAFE-400 reactor core, a tally of the secondary neutron current was obtained at the core-reflector interface, yielding a net current of $1.4 \times 10^{7} \mathrm{n} / \mathrm{s}$ due to the space radiation environment at geosynchronous orbit in the presence of the 1989 solar particle event (peak 5-min flux). During steady-state full power operation, a $400 \mathrm{kWt}$ reactor produces approximately $10^{16} \mathrm{n} / \mathrm{s}$. Typical terrestrial reactor start-up sources are on the order of $10^{6}-10^{8} \mathrm{n} / \mathrm{s}$ (Knoll, 1983). Hence, the secondary neutron source would not be significant during full power operation, but these results do suggest that the natural radiation environment could provide a reliable start-up source.

Because the location of the secondary neutron source is significant to its importance, the core leakage current does not provide conclusive evidence that the reactor would reliably start. An additional MCNPX run was performed to compute the flux across the reactor core that results from the external proton source striking the reactor in 4-pi geometry. Fig. $3(b)$ shows the neutron flux in a subcritical SAFE- $400\left(k_{\text {eff }}=0.97695 \pm 0.00022\right)$, revealing the expected cosine shape that corresponds to the fundamental mode. The flux level of $10^{5} \mathrm{n} / \mathrm{cm}^{2} \mathrm{~s}$ is more than 
sufficient to start the reactor. For comparison purposes, a fission source was placed in the same subcritical geometry. The resulting neutron flux is shown in Fig. 3(c). The similarity in the flux shape for the reactor started by the external radiation source and the one started by a typical fission neutron distribution is readily evident, although the magnitude of the flux is considerably greater in the latter simulation. To clarify the similarity in the neutron flux in each simulation, the results along the central cross section of the reactor were normalized to the maximum neutron flux in the central fuel pin for each simulation. The normalized curves plotted in Fig. 4 demonstrate that the external radiation source indeed provides a neutron flux distribution identical to the reactor started using a fission source.

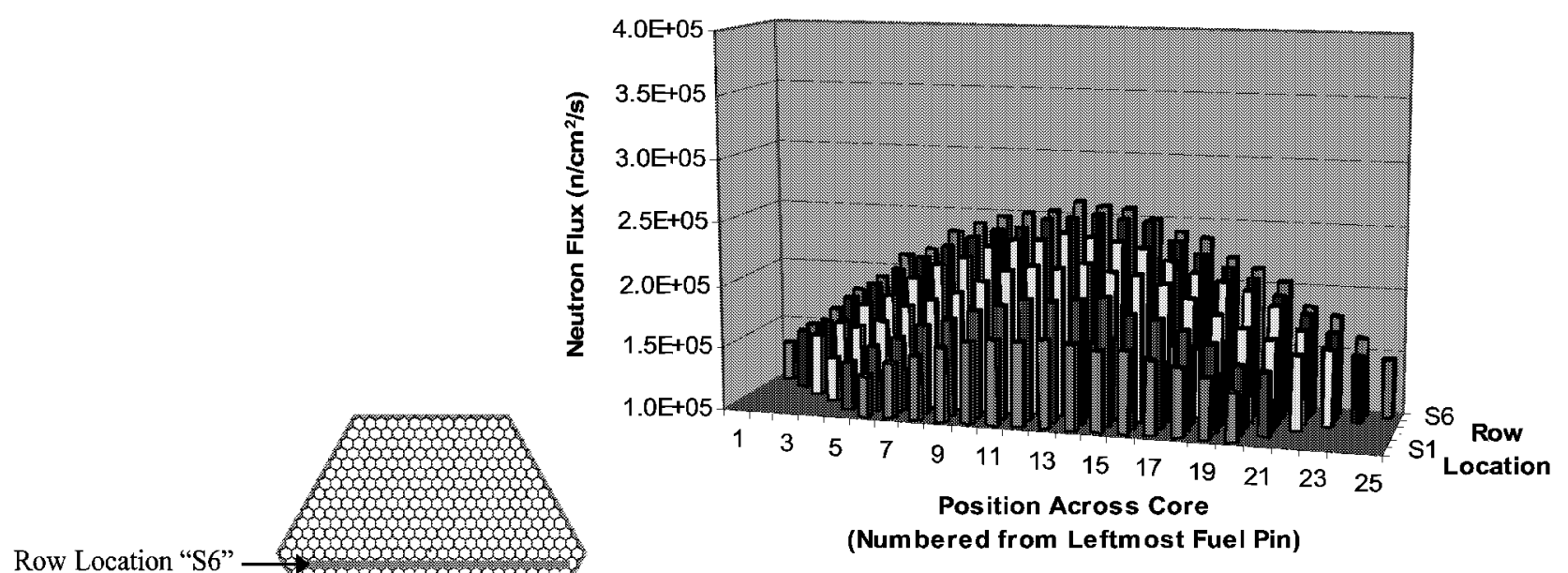

(b)
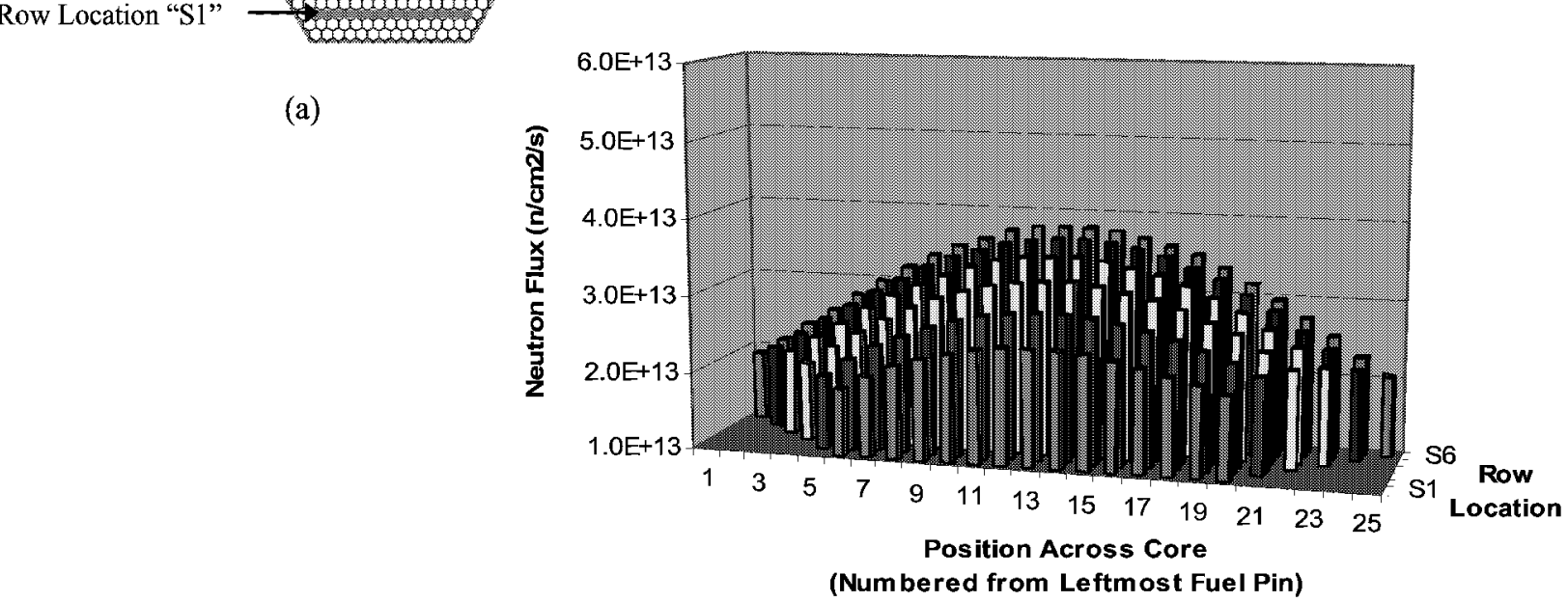

(c)

FIGURE 3. Reactor Neutron Flux for keff $=0.97695 \pm 0.00022$. (a) Simplified Reactor Cross Section Identifying Locations of Each Series Plotted in (b) and (c). (b) Neutron Flux Due to External Proton Source. (Uncertainty for all values is 15\%.) (c) Reactor Neutron Flux Due to a Fission Source. (Uncertainty for all values is less than 1\%.)

\section{Results of Optimized Control Algorithm}

Results of the control algorithm described in Eqs. (1-5) are provided in Fig. 5. A power level of $1 \mathrm{kWt}$ was selected as appropriate power level to initialize the reactor prior to full system operation at the nominal power level of 400 $\mathrm{kWt}$. Startup to the selected power level is accomplished in under 5 seconds with minimal power overshoot. At this power level, heat pipe thaw can begin and the power conversion system can be initiated as the power level is further 


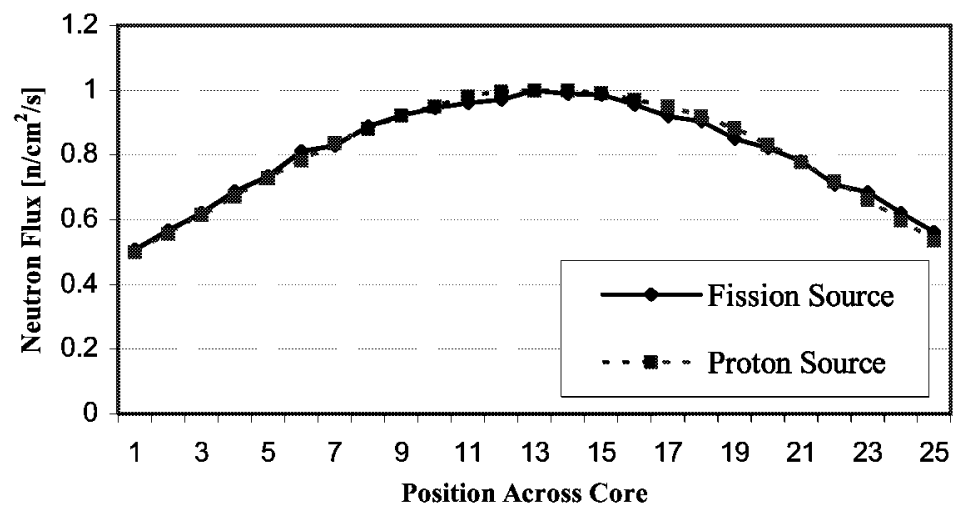

FIGURE 4. Comparison of Normalized Flux Level Along the Central Reactor Cross Section (Row "S6" shown in Fig. 3(a)) for the Externally-Started Reactor and the Reactor Started Using a Fission Source.

increased. The optimized control vector for the rate of rotation of the control drums is shown on the left-hand side of Fig. 5, as well as the resulting angular position of the control drums. The final position of the control drums is 89.84 degrees. The result shown here corresponds to a constant external source of $10^{6}$ neutrons $/ \mathrm{sec}$, a nominal background level for operation in the space radiation environment. For computational purposes, the prompt neutron lifetime is assumed to be $10^{-3} \mathrm{sec}$ in the simulation presented here; this value will be adjusted to the more appropriate lifetime for a fast reactor $\left(\sim 10^{-7} \mathrm{sec}\right)$ in subsequent versions of the control algorithm.

Two separate power trajectories are shown in Fig. 5(b) and 5(d). The trajectory in Fig. 5(d) corresponds to the reactor power when the control is applied in a closed loop. The optimal control step is re-calculated at each sampling interval, and the control vector is modified to the appropriate value based on the present conditions in the plant. The trajectory in Fig. 5(b) corresponds to an open loop system. The optimal control vector pre-determined in the closed loop program was applied in a single step to the reactor system, but without the feedback provided in closed-loop control, the plant has no mechanism to make adjustments. The small numerical differences that result from solving the PKE on a short $(\sim 1 \mathrm{sec})$ versus a long $(\sim 100 \mathrm{sec})$ time scale cause the "after-the-fact" computation, utilizing a pre-determined control vector, to drift away from the desired power level because there is no active controller to push it back to the appropriate value. The open-loop system is slightly unstable, so the errors accumulate and the system drifts away from the desired power. This result clearly demonstrates the superior performance of the system when under feedback control.

The same control methodology applied above, in the presence of a constant background source, was also applied for a time varying source. As shown in Fig. 6, the source was perturbed at times 3, 10, and 50 seconds after initializing startup procedures. The initial perturbation during the power rise causes a slight shift in the power (evident only when looking at reactor power over the early part of the control horizon), but the controller quickly recovers and approaches the goal of $1 \mathrm{kWt}$ in approximately the same time as in the previous simulation. After achieving steady state power at $1 \mathrm{kWt}$, source perturbations have no effect on the reactor power because the neutron production rate in the reactor core is on the order of $10^{14} \mathrm{n} / \mathrm{s}$ while the neutron source strength never exceeds $10^{9} \mathrm{n} / \mathrm{s}$. Hence, the controller proposed here is sufficiently robust to maintain reactor control in the presence of source fluctuations. Simulation of reactor control with shorter time increments (a control step of $1 \mathrm{sec}$ was applied in the present implementation) may lend further insight to the reactor behavior in the presence of a time varying source.

\section{CONCLUSIONS}

The space radiation environment can provide sufficient neutron production to preclude the necessity of a source for reactor startup and can be of sufficient magnitude to affect reactor operations at low power. Results for the sample space radiation environment in geosynchronous Earth orbit generated a reactor neutron flux on the order of $10^{5}$ $\mathrm{n} / \mathrm{cm}^{2} \mathrm{~s}$ and a corresponding leakage current on the order of $10^{7} \mathrm{n} / \mathrm{s}$, comparable to typical reactor start-up sources. 


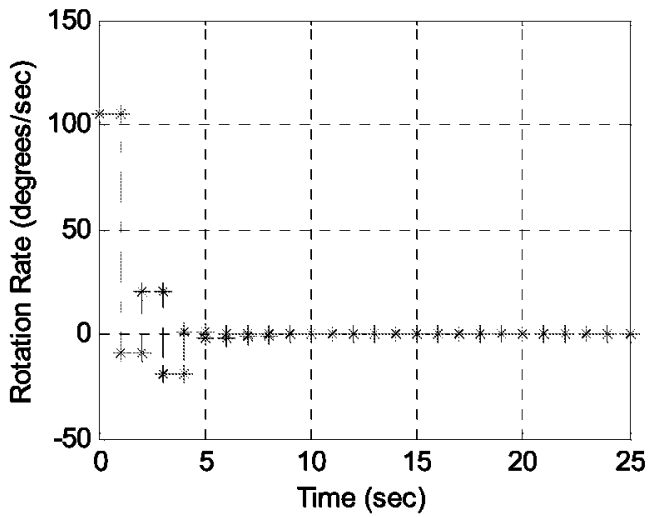

(a) Optimized Control Vector

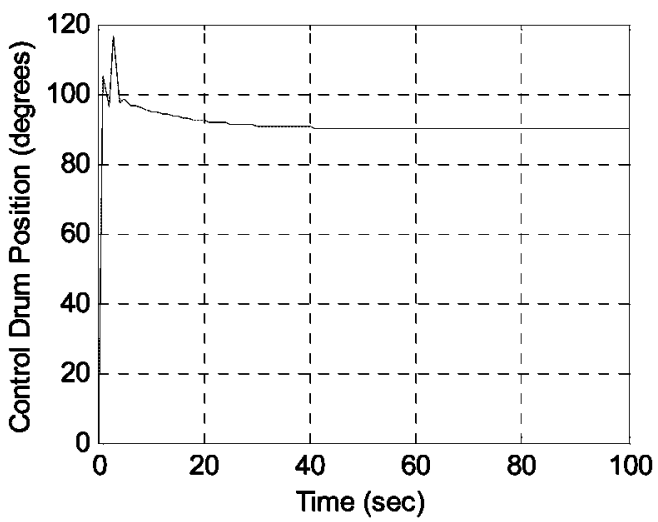

(c) Angular Position of Control Drums

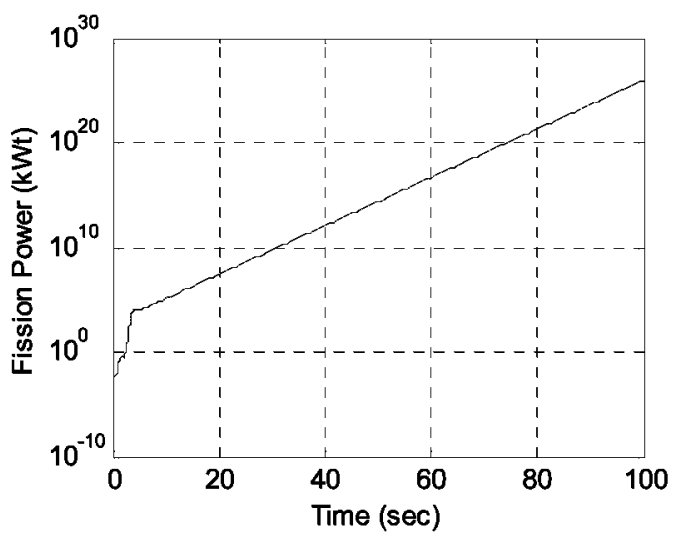

(b) Open Loop Control

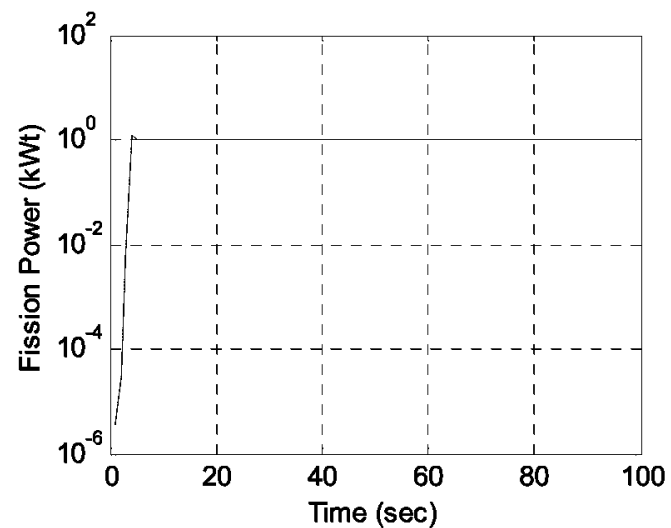

(d) Closed Loop Feedback Control

FIGURE 5. Sample Reactor Startup Control Program to $1 \mathrm{kWt}$. (Note that the control vector is shown over a shorter time scale of $25 \mathrm{sec}$ to allow the detail at early times to be observed.) Plots (b) and (d) demonstrate the benefit of closed loop control.

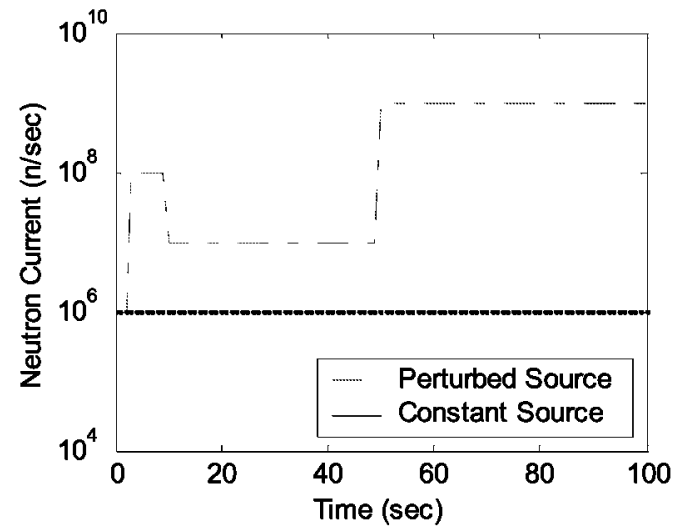

(a) Extemal Neutron Source

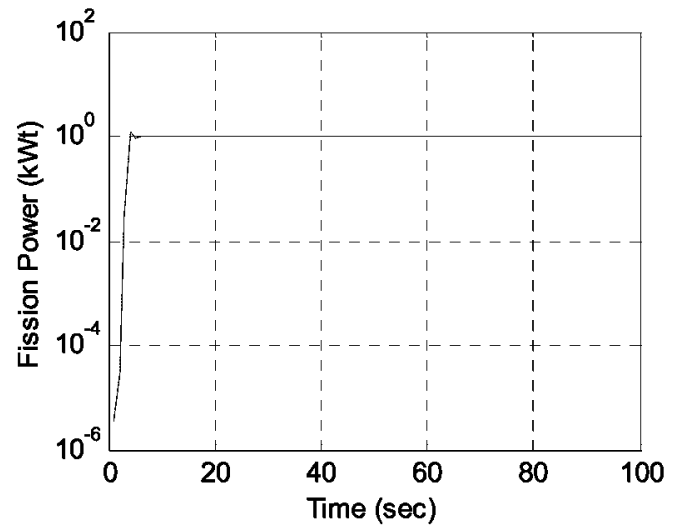

(b) Reactor Power, Closed Loop Feedback Control

FIGURE 6. Controller Operation and Resulting Reactor Power in the Presence of Source Perturbations.

Because a possible mission for an NEP vehicle is to the Jovian system, future work will analyze the reactor response in the presence of the intense Jovian radiation environment, where the particle fluence can be orders of magnitude greater than in near-Earth space (Jun et al., 2002). Although low power operations are not be anticipated in the Jovian system, the potential response of the reactor in this radiation environment should be characterized should low 
power transients be necessary. Future work will also consider the additional neutron population generated by the $\mathrm{Z} \geq 2$ particles in the radiation environment. Because these components exist at very high energies, it is anticipated that they will cause significant spallation neutrons to be produced, increasing the background radiation environment in which the reactor must operate.

In an environment having a noisy external radiation source, considerable design efforts must go into constructing autonomous start-up and control procedures. The MBPC methodology presented here demonstrates that stable reactor operation with minimal overshoot of the desired power can be obtained in a very short time if robust feedback control is applied, in the presence of either a constant or fluctuating source. This control program will serve as a basis for future studies of reactor operation in more intense, highly variable radiation environments.

\section{NOMENCLATURE}

$\beta=$ delayed neutron fraction

$\lambda_{i}=$ decay constant for the $i^{\text {th }}$ type precursor $\left(\sec ^{-1}\right)$

$\Lambda=$ prompt neutron lifetime (sec)

$v=$ number of neutrons produced per fission

$\rho=$ reactivity

$\theta=$ angular position of control drums (degrees)

$\omega=$ rate of rotation of control drums (degrees/sec)

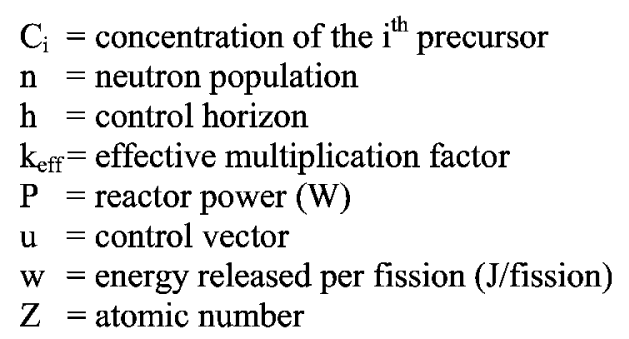

$C_{i}=$ concentration of the $i^{\text {th }}$ precursor

n

$\mathrm{k}_{\mathrm{eff}}=$ effective multiplication factor

$\mathrm{P}=$ reactor power $(\mathrm{W})$

$\mathrm{w}=$ energy released per fission $(\mathrm{J} /$ fission $)$

$\mathrm{Z}=$ atomic number

\section{ACKNOWLEDGMENTS}

This work was supported in part by the Propulsion Research Center at the NASA Marshall Space Flight Center, as a part of the Graduate Student Researchers Fellowship Program. The second author (JPH) was supported in part by DOE grant DE-FG07-02SF22612.

\section{REFERENCES}

Clarke, D.W., "Advances in Model-Based Predictive Control," in Advances in Model-Based Predictive Control, edited by D.L. Clarke, Oxford University Press, Inc., New York, 1994, pp. 3-21.

Feynman, J. and Gabriel, S. B., "High-energy charged particles in space at one astronomical unit," IEEE Transactions on Nuclear Science, 43(2), pp. 344-352 (1996).

Hetrick, D.L., Dynamics of Nuclear Reactors, the University of Chicago Press, Chicago, 1971.

Jun, I., Ratliff, J.M., Garrett, H.B., McEntire, R.W., "Monte Carlo Simulations of the Galileo Energetic Particle Detector," Nuclear Instruments \& Methods in Physics Research Section A: Accelerators Spectrometers Detectors and Associated Equipment 490(3), pp. 465-475, 2002.

Knoll, G.F., "Radioisotope Neutron Sources," in Neutron Sources for Basic Physics and Applications, edited by S. Cierjacks, Pergamon Press, 1983, pp. 7-18.

Lagarias, J.C., Reeds, J.A., Wright, M.H., and Wright, P.E., "Convergence Properties of the Nelder-Mead Simplex Method in Low Dimensions," SIAM Journal of Optimization, Vol. 9(1), pp. 112-147 (1998).

Morari, M., "Model Predictive Control: Multivariable Control, Technique of Choice in the 1990s?," in Advances in Model-Based Predictive Control, edited by D.L. Clarke, Oxford University Press, Inc., New York, 1994, pp. 22-37.

Naval Research Laboratory, "Cosmic Ray Effects on MicroElectronics," (1996), http://crsp3.nrl.navy.mil/creme96/, accessed April 2003.

Poston, D.I., Kapernick, R.J., and Guffee, R.M., "Design and Analysis of the SAFE-400 Space Fission Reactor," in proceedings of Space Technologies and Applications International Forum (STAIF-2002), edited by M. El-Genk, AIP Conference Proceedings 608, New York, 2002, pp. 578-588.

Tylka, A.J., Adams, J. H., Boberg, P. R., Brownstein, B., Dietrich, W. F., Flueckiger, E. O., Petersen, E. L., Shea, M. A., Smart, D. F., and Smith, E. C., "CREME96: A Revision of the Cosmic Ray Effects on Micro-Electronics Code," IEEE Transactions on Nuclear Science, 44(6), pp. 2150-2160 (1997).

Waters, L.S., MCNPX User's Manual, Version 2.3.0, April 2002, LA-UR-02-2607, 2002.

Wilson, J.W., Cucinotta, F.A., Tai, H., Simonsen, L.C., Shinn, J.L., Thibeault, S.A., and Kim, M.Y., "Galactic and Solar Cosmic Ray Shielding in Deep Space," NASA Technical Paper 3682, 1997. 